\title{
Late Marek's Disease in Adult Chickens Inoculated with Virulent Marek's Disease Virus
}

\author{
Mitsutaka IKEZAWA ${ }^{1,2)}$, Masanobu GORYO ${ }^{1,2) *}$, Jun SASAKI ${ }^{2)}$, Mohie HARIDY ${ }^{1)}$ and Kosuke OKADA ${ }^{1,2)}$ \\ ${ }^{1)}$ Department of Pathogenic Veterinary Science, The United Graduated School of Veterinary Sciences, Gifu University, 1-1 Yanagido, \\ Gifu 501-1193 and ${ }^{2}$ Department of Veterinary Pathology, Faculty of Agriculture, Iwate University, 3-18-8 Ueda, Iwate 020-8550, Japan
}

(Received 13 May 2010/Accepted 7 July 2010/Published online in J-STAGE 21 July 2010)

ABSTRACT. Recently, excessive losses from Marek's disease (MD) have been noted in adult laying flocks over the age of 40 weeks. We defined these late outbreaks in adult chickens as "late MD", and experimentally reproduced the disease in adult SPF P2 line (50-weekold) or commercial line (74-week-old) chickens inoculated with a virulent strain of Marek's disease virus (MDV). Commercial line chickens were given MDV vaccines (HVT and CVI 988) at hatch. The occurrence of MD was evaluated periodically by the evidence of neurologic signs such as paralysis, torticollis, ataxia, and/or nervous tics, as well as histopathological examination. In P2 line chickens, neurologic signs and MD lymphoma were observed from day 21 onward, and they tended to increase in a time-dependent manner. Meanwhile, in commercial line chickens, only one chicken exhibited MD lymphoma on day 70 post inoculation, but its pathogenesis was questionable. No regression of MD lymphoma was noted in either case. The lesions in the visceral organs, thymus, peripheral nerves, and feather pulps of P2 line chickens were characterized by proliferation of variably sized lymphoid cells. In the feather follicle epithelium, numerous inclusion bodies were noted on day 21 post-inoculation, which tended to decrease afterwards. The morphological findings obtained resembled late MD in field cases. In conclusion, our results demonstrate that adult SPF P2 line chickens are susceptible to virulent MDV, and would be useful for investigation of late MD.

KEY WORDS: adult chickens, experimental reproduction, histopathology, late Marek's disease.

J. Vet. Med. Sci. 72(12): 1539-1545, 2010

Marek's disease virus (MDV), currently reclassified as Gallid herpesvirus 2, is an $\alpha$-herpesvirus belonging to the genus Mardivirus and the family Herpesviridae. MDV infection in chickens causes Marek's disease (MD), a lymphoproliferative and neurologic disease, resulting in the formation of malignant $\mathrm{T}$ cell lymphomas $[3,17]$.

Generally, MD has been thought to be a disease for young chickens ( $c a .4$ to 20 weeks old), but, recently excessive losses from MD have been noted in adult laying flocks over the age of 40 weeks [21]. It was reported that MD frequently occurred after molting in preparation for a second lay cycle $[11,12,21]$. Moreover, MD outbreaks in adult chickens were reported to become very common recently [16]. In this study, we defined these late MD outbreaks in adult chickens as "late MD" in contrast with conventional "early MD" in young chickens.

In commercial poultry industries, MD is usually prevented by vaccination, which inhibits the onset of tumor formation, but not that of virus infection on its own. The mechanism of tumor prevention by vaccination still remains unclear $[2,5,15]$. If the protective immunity against MD was not established after vaccination, chickens would develop MD at younger ages, and consequently die. For instance, co-infection with immunodepressive pathogens such as chicken anemia virus enhanced MD outbreaks in both young and adult flocks $[7,16,19]$. Thus, age or infectious stresses were considered to be predisposing factors in

\footnotetext{
* CoRrespondence to: Goryo, M., Department of Veterinary Pathology, Faculty of Agriculture, Iwate University, 3-18-8 Ueda, Iwate 020-8550, Japan.

e-mail: goryo@iwate-u.ac.jp
}

late MD outbreaks [18, 21].

So-called "age resistance" which is regarded as an expression of genetic resistance is best demonstrated in SPF chickens $[4,21]$. In most experimental studies, the combination of SPF chickens aged 12 to 22 weeks and virulent MDV strains has been well utilized [1, 4, 20, 23], although they were considerably young compared to the ages eliciting late MD outbreaks in field flocks. There have been few reports addressing the susceptibility of adult chickens $(>30$ week-old) to MD. It is essential to establish an appropriate experimental model of late MD for elucidating its pathogenesis. In the present work, adult SPF P2 line (50-week-old) or commercial line (74-week-old) chickens were inoculated with virulent MDV for an attempt to reproduce late MD experimentally and assessed them neurologically and histopathologically.

\section{MATERIALS AND METHODS}

Chickens: Twenty-five adult SPF P2 line (genetically MD-susceptible) chickens aged 50 weeks were in-bred at the Laboratory of Veterinary Pathology, Iwate University (Morioka, Japan). They were derived from White Leghorn line P2 flocks. The chickens were free from antibody to adenovirus, avian infectious bronchitis virus, chicken anemia virus, infectious bursal disease virus, MDV, Newcastle disease virus, reovirus, avian leukosis virus, and other avian pathogens. They were reared in a small isolated room from hatch and supplied food (Chubushiryo Co., Ltd., Nagoya, Japan) and tap water ad libitum. Twenty adult commercial line chickens aged 74 weeks were provided kindly from a 
commercial farm. They are a genetically MD-resistant line. The commercial line chickens were inoculated with MD vaccines (HVT and CVI 988, Ghen Co., Ltd., Gifu, Japan) at hatch, and reared in the field environment.

All chicken were reared and manipulated in accordance with the Guidelines for Animal Experimentation issued by the Japanese Association for Laboratory Animal Science [10] and also approved by the Animal Experimental Ethics Committee of Iwate University (Morioka, Japan).

Virus: Virulent KS strain [9, 18] of oncogenic serotype 1 was used as the MDV in this study. This strain is equivalent to the JM strain. The inoculum contained virulent MDV at titer of 3,000 PFU/0.1 ml.

Polymerase chain reaction (PCR): Before the experiment, expression of the MDV genome in each chicken was investigated by PCR. Briefly, total DNA was extracted from the tip of growing wing feathers as described by Davison and Borenshtain [6] with some modifications. The feather tips were chopped and incubated overnight in lysis buffer at $55^{\circ} \mathrm{C}$, purified by phenol/chloroform, precipitated with ethanol, and dissolved in $\mathrm{T}_{10} \mathrm{E}_{1}$ buffer. The primers (M1 and M2) were designed to detect the 132 base pair tandem repeat (BamH1-H,D genomic fragments).

M1: (5'-TACTTCCTATATAGATTGAGACCGT-3')

M2: (5'-GAGATCCTCGTAAGGTGTAATATA-3')

The primers were synthesized by SIGMA-Aldrich Japan Co., Ltd. (Tokyo, Japan). The PCR was carried out using a Thermal Cycler PERSONAL (TaKaRa Bio Co., Ltd., Otsu, Japan), and performed by the procedure [6] as described previously.

Experimental designs: Twenty P2 line chickens (chicken Nos. S1 to S20) were inoculated intramuscularly at the femoral region with $0.1 \mathrm{~m} l$ of inoculum. The inoculation day was regarded as day 0 . Each groups of five chickens were euthanized by exsanguination under ether anesthesia on days 21 (Nos. S1 to S5) and 42 (Nos. S7 to S11). During the experiment periods, two chickens were dead and necropsied upon discovery on days 24 (No. S6) and 54 (No. S12), and four moribund chickens were found and euthanized under ether anesthesia on days 56 (Nos. S13 and S14) and 66 (No.S15 and S16). At termination on day 70, 4 surviving chickens (Nos. S17 to S20) were euthanized by the aforementioned procedure. Five control chickens (Nos. S21 to S25) received a $0.9 \%$ physiological saline solution on day 0 , and were euthanized on day 70 .

Fifteen commercial line chickens (Nos. C1 to C15) were inoculated intramuscularly at the femoral region with $0.1 \mathrm{ml}$ of inoculum. Five chickens (Nos. C1 to C5) were euthanized on day 21, and ten remaining chickens (Nos. C6 to C15) were euthanized on day 70 . Five control chickens (Nos. C16 to $\mathrm{C} 20$ ) received a $0.9 \%$ physiological saline on day 0 , and were euthanized on day 70 .

Neurologic signs: Neurologic signs including paralysis, torticollis, ataxia, and/or nervous tics were scored according to the observation battery of Gimeno et al. [8] with minor modifications.

Histopathological examination: After gross examination of dead, moribund, and schedule-killed chickens, the visceral organs (liver, spleen, kidney, heart, and lung), peripheral nerves, skin, lymphoid organs (thymus and cecal tonsil), and other affected organs were removed, fixed in $10 \%$ formalin and embedded with a routine process in paraffin-wax. All sections were cut approximately $4 \mu \mathrm{m}$ thick and stained with hematoxylin and eosin (HE). The sections were histopathologically examined using an optical microscope. The severity of MD lymphomas in the affected organs and the histological lesions in the peripheral nerve and skin was scored as $(-)$ to $(3+)$ and $(-)$ to $(4+)$, respectively, under the criteria as shown in the foot notes of corresponding Tables 1 and 2 .

\section{RESULTS}

Identification of MDV infection by PCR: Expression of the MDV genome in P2 line and commercial line chickens was confirmed to be negative and positive, respectively, prior to the experiments.

Neurologic signs and MD lymphoma: In P2 line chickens (Table 1), one (No. S4) had renal lymphoma without neurologic signs on day 21. On day 42 , three (Nos. S8, S9, and S11) displayed neurologic signs, while one (No. S8) of them exhibited renal lymphoma. On days 54 to 66, five (Nos. S12 to S16) died or were euthanized due to poor health, preceded by apparent neurologic signs. Three (Nos. S12, S13, and S15) of them had lymphoma in the visceral organs except the spleen. At termination on day 70, four (Nos. S17 to S20) survived and two of them (Nos. S18 and S19) showed neurologic signs with or without lymphoma in the multiple organs. The incidence of neurologic signs and visceral MD lymphoma was $50 \%(10 / 20)$ and $30 \%(6 / 20)$, respectively. During days 42 and 70, the incidence of neurologic signs and visceral MD lymphoma was $71.4 \%(10 / 14)$ and $35.7 \%$ $(5 / 14)$, respectively.

In commercial line chickens, neither neurologic signs nor gross lymphomas were observed throughout the experimental period (data not shown). However, one (No. C4) had a pulmonary MD lymphoma, diagnosed by the histopathological examination as mentioned below.

No clinicopathological abnormalities were seen in the corresponding control chickens.

Histopathological findings: Detailed histopathological findings for individual P2 line chickens are summarized in Tables 1 and 2. In MD lymphomas of the visceral organs and thymus, moderate to massive proliferation of variably sized lymphoid cells was observed sporadically throughout the experimental period.

In the peripheral nerves, small foci of lymphoid cells (Fig. 1A) with or without inflammatory lesions were noted on day 21. On day 42, minimal to diffuse proliferation of variably sized lymphoid cells and scant to severe neuritis with infiltration of lymphocytes, plasma cells, heterophils and macrophages were observed (Fig. 1B). Additionally, various degrees of edema, necrosis, and axon degeneration were commonly noted. On days 54 to 66, diffuse lymphoid 
Table 1. Neurologic signs (NS) and MD lymphoma of the visceral organs and thymus in adult SPF P2 line chickens inoculated with virulent MDV

\begin{tabular}{|c|c|c|c|c|c|c|c|c|}
\hline \multirow{2}{*}{$\mathrm{DPI}^{\mathrm{a})}$} & \multirow{2}{*}{$\begin{array}{c}\text { Chicken } \\
\text { No. }\end{array}$} & \multirow{2}{*}{ NS } & \multicolumn{6}{|c|}{ Organs } \\
\hline & & & Liver & Spleen & Kidney & Heart & Lung & Thymus \\
\hline \multirow{5}{*}{$21^{d)}$} & S1 & -b) & _c) & - & - & - & - & - \\
\hline & S2 & - & - & - & - & _- & _- & _- \\
\hline & S3 & - & _- & - & _- & - & - & _- \\
\hline & S4 & - & - & - & $2+$ & - & - & - \\
\hline & S5 & - & - & - & - & - & - & - \\
\hline $24^{\mathrm{e})}$ & S6 & - & - & - & - & - & - & - \\
\hline \multirow{5}{*}{$42^{\mathrm{d})}$} & S7 & - & - & - & - & - & - & - \\
\hline & S8 & + & - & - & $3+$ & - & - & - \\
\hline & S9 & + & - & - & - & - & - & - \\
\hline & S10 & - & - & - & - & - & - & - \\
\hline & S11 & + & - & - & - & - & - & - \\
\hline $54^{\mathrm{e})}$ & $\mathrm{S} 12$ & + & - & - & $3+$ & $3+$ & - & $3+$ \\
\hline \multirow{2}{*}{$56^{\mathrm{e})}$} & $\mathrm{S} 13$ & + & - & - & - & - & $2+$ & $3+$ \\
\hline & S14 & + & - & - & - & - & - & - \\
\hline \multirow{2}{*}{$66^{\mathrm{e})}$} & S15 & + & $3+$ & - & - & - & - & - \\
\hline & S16 & + & - & - & - & - & - & - \\
\hline \multirow{4}{*}{$70^{\mathrm{d})}$} & S17 & - & - & - & - & - & - & - \\
\hline & $\mathrm{S} 18$ & + & $3+$ & - & $3+$ & $3+$ & $2+$ & - \\
\hline & $\mathrm{S} 19$ & + & - & - & - & - & - & - \\
\hline & S20 & - & - & - & - & - & - & - \\
\hline
\end{tabular}

a) DPI=days post inoculation.

b) -; no abnormal signs, +; incidence of paralysis, torticollis, ataxia and/or nervous tics.

c) -; no abnormal lesion, +; small accumulation of lymphoid cells, 2+; moderate proliferation or mass formation of lymphoid cells, $3+$; massive proliferation or mass formation of lymphoid cells disrupting the normal architectures.

d) schedulled euthanasia.

e) death or euthanized becouse of the moribund conditions.

cell proliferation was seen (Fig. 1C), but inflammatory lesions were not identified because of marked neoplastic lesions. On day 70, slight lymphocyte infiltration was observed with or without focal neoplastic lesions and edematous nerve fibers (Fig. 1D).

In the skin, numerous inclusion bodies were seen in the feather follicle epithelium (Fig. 2) with slight neoplastic and inflammatory lesions on day 21 . On day 42 , moderate to diffuse lymphoid cell proliferation in the feather pulps was observed in association with a moderate number of inclusion bodies in the feather follicle epithelium and slight to moderate inflammatory lesions around the feather follicles. On days 54 and 56, severe lymphoid cell proliferation with various degrees of inflammatory cell infiltration was observed with moderate inclusion bodies. On days 66 and 70 , slight lymphocyte infiltration or severe lymphoid cell proliferation was noted with only a few inclusion bodies.

In commercial line chickens, a small number of inclusion bodies was observed in the feather follicle epithelium on day 21 , but not thereafter. On days 21 to 70 , only a few lymphocytes were observed around blood vessels in the peripheral nerves (Fig. 3). On day 70, only one chicken (No. C4) showed lymphoma containing variably sized lymphoid cells in the lung and skin (Fig. 4). No neoplastic or inflammatory lesions were seen in other chickens throughout the experimental period.

No histopathological lesions suggesting regression of MD lymphoma were noted in either case.

\section{DISCUSSION}

Susceptibility to MDV is considered to be much lower in older chickens than in younger chicks. Although the "ageresistance" remained unclear, the immune system maturation likely participated in this event. Witter et al. [22] reported that vaccinated-susceptible SPF line chickens (18week-old) did not cause MD regardless of infection with a highly virulent strain $\left(\mathrm{vv}\right.$ and $\left.\mathrm{vv}^{+}\right)$of $\mathrm{MDV}$. In the field flocks, although chicks receiving vaccination were exposed to environmentally resident MDV during their lives, only individuals bearing a proper immune response exerted a protective action against MD. Conversely, adult chickens with poor immune responses including SPF were considered to possess at least in part a high susceptibility to late MD. In our work using SPF P2 line chickens (50-weekold), MD developed from day 21 onward, and a total of six chickens were found dead or moribund during the experimental period. 
Table 2. Histopathological findings of the peripheral nerves and skins in adult SPF P2 line chickens inoculated with virulent MDV

\begin{tabular}{|c|c|c|c|c|c|c|c|c|}
\hline \multirow{3}{*}{$\mathrm{DPI}^{\mathrm{a})}$} & \multirow{3}{*}{$\begin{array}{c}\text { Chicken } \\
\text { No. }\end{array}$} & \multicolumn{2}{|c|}{ Peripheral nerves } & \multicolumn{5}{|c|}{ Skins } \\
\hline & & \multirow{2}{*}{ Neoplastic lesion } & \multirow{2}{*}{ Inflammation } & \multicolumn{2}{|c|}{ Feather pulp } & \multicolumn{3}{|c|}{ Feather follicle } \\
\hline & & & & Neoplastic lesion & Inflammation & IB & Neoplastic lesion & Inflammation \\
\hline \multirow{5}{*}{$21^{\mathrm{c})}$} & S1 & +b) & - & - & + & $3+$ & - & + \\
\hline & S2 & + & + & - & + & $3+$ & $2+$ & + \\
\hline & S3 & + & + & $\mathrm{NE}$ & $\mathrm{NE}$ & $3+$ & - & - \\
\hline & S4 & + & - & - & + & $3+$ & - & - \\
\hline & S5 & - & - & - & + & $3+$ & - & - \\
\hline $24^{\text {d) }}$ & S6 & - & + & $\mathrm{NE}$ & & - & - & + \\
\hline \multirow{5}{*}{$42^{\mathrm{c})}$} & S7 & + & + & - & + & $2+$ & - & $2+$ \\
\hline & S8 & + & $2+$ & $\mathrm{NE}$ & $\mathrm{NE}$ & $\mathrm{NE}$ & $\mathrm{NE}$ & $\mathrm{NE}$ \\
\hline & S9 & $3+$ & $2+$ & - & + & - & + & $2+$ \\
\hline & S10 & + & + & $3+$ & ND & $2+$ & - & $2+$ \\
\hline & S11 & $2+$ & + & $2+$ & + & $2+$ & + & + \\
\hline $54^{\mathrm{d})}$ & S12 & $2+$ & ND & $3+$ & ND & $2+$ & $2+$ & + \\
\hline \multirow{2}{*}{$56^{\mathrm{d})}$} & S13 & $3+$ & ND & $3+$ & ND & $2+$ & $4+$ & ND \\
\hline & S14 & $3+$ & ND & - & + & + & - & + \\
\hline \multirow{2}{*}{$66^{\mathrm{d})}$} & S15 & $4+$ & ND & - & + & - & - & + \\
\hline & S16 & $4+$ & ND & - & + & + & - & + \\
\hline \multirow{4}{*}{$70^{\mathrm{c})}$} & S17 & - & - & - & - & - & - & - \\
\hline & S18 & $2+$ & + & $4+$ & ND & + & $3+$ & ND \\
\hline & S19 & - & + & - & - & + & - & + \\
\hline & S20 & - & + & - & - & - & - & $2+$ \\
\hline
\end{tabular}

a) DPI=days post inoculation.

b) Neoplastic lesions, -; no abnormal lesions, +; slight proliferation of lymphoid cells around vessels, 2+; moderate proliferation of lymphoid cells, 3+; diffuse proliferation of lymphoid cells, 4+; massive diffuse proliferation of lymphoid cells.

Inflammation, -; no abnormal lesions, +; slight infiltration of inflammatory cells, 2+; moderate accumulation or infiltration of inflammatory cells, $3+$; diffuse infiltration of inflammatory cells, $4+$; massive infiltration of inflammatory cells.

$\mathrm{IB}=$ inclusion bodies. Numbers of inclusion bodies in the feather follicle epithelium were scored as follows, -; no abnormal lesion, +; low, 2+; moderate, $3+$; severe.

$\mathrm{NE}=$ not examined. $\mathrm{ND}=$ not diagnosed because of severe neoplastic lesions.

c) schedulled euthanasia.

d) death or euthanized because of the moribund conditions.

In previous studies of virulent strain MDV infection [1, 4, 20], chickens aged 12 to 22 weeks served as an adult chicken model, because avian immune system became established by 4 to 6 weeks of age. Under these experimental conditions, MD was induced between 16 and 32 weeks. In contrast, late MD in the field flocks was recognized to occur mainly at $60-80$ weeks [21]. Thus, there have been considerable gaps of age in chickens between the results of these experimental and field studies. Since our aim is to examine the reproduction of late MD in field flocks, we selected adult SPF chickens aged 50 weeks old and commercial line field (74 weeks old) for our current investigation.

The incidence of neurologic signs and visceral MD lymphoma in the P2 line between days 21 and 70 was 55 and $30 \%$, respectively, which were relatively higher than that in previous reports $[1,4,20]$. Differences in the incidence may have resulted from host-virus interactions. In a study using SPF susceptible line chickens (18-week-old) inoculated with virulent (JM/102W, GA/22, 617A, or RB1B) MDV by Witter et al. [22], the occurrence of MD by 63 days postinoculation was $79-100 \%$ for the strain $\mathrm{JM} / 102 \mathrm{~W}$, and $7-$
$21 \%$ for strains $\mathrm{GA} / 22,617 \mathrm{~A}$, and RB1B strains. Taken together, it is probable that adult chickens, bearing the same genetic background, might elicit a different response, when inoculated with different strains of virulent MDV. The incidence of MD may be attributable to interactions between the genetic background in adult chickens and the genetic diversity of the used MDV. In P2 line chickens, the incidence of neurologic signs and MD lymphoma between days 42 and 70 was 71.4 and $35.7 \%$, respectively, suggesting that they were likely to be more susceptible to virulent MDV KS strain. It is surmised that the SPF P2 line is still susceptible to virulent MDV infection even at older ages.

Histopathologically, the lesions in the visceral organs, thymus and peripheral nerves were characterized by proliferation of variably sized lymphoid cells, resembling MD in adult chickens [20, 22], as well as late MD in our field cases [unpublished data]. In the feather follicle epithelium, numerous inclusion bodies were noted on day 21 , but these alterations tended to decrease thereafter. Our findings were supported by the data from younger P2 line chicks $[9,18]$. To the best of our knowledge, no reports have dealt with between initial inclusion body formation and later MD 

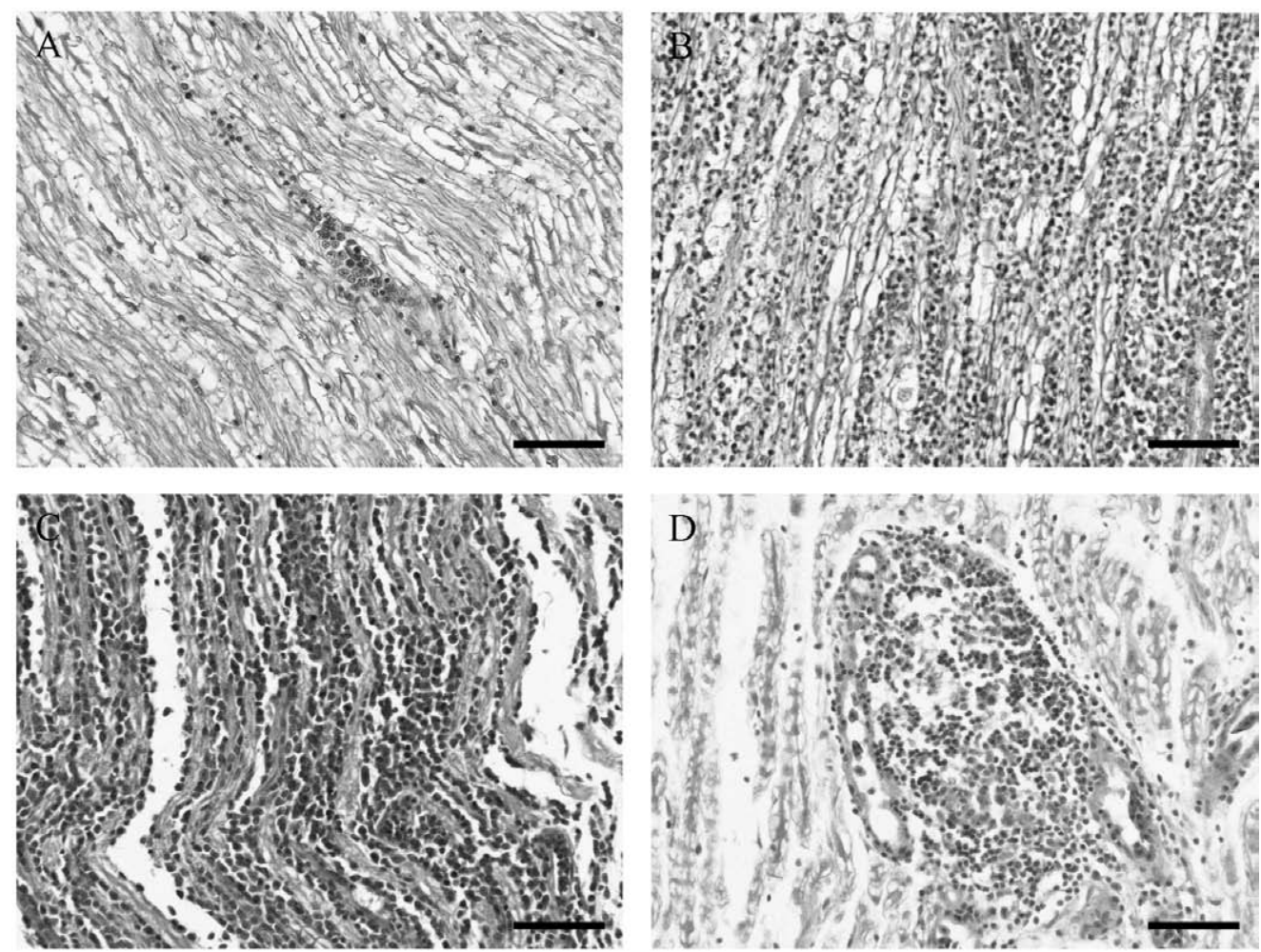

Fig. 1. Inflammatory and neoplastic lesions in the peripheral nerve of adult SPF P2 line chickens (50-week-old) inoculated with virulent MDV. (A) Small foci of variably sized lymphoid cells in a chicken (No. S4) on day 21. (B) Diffuse proliferation of variably sized lymphoid cells and severe neuritis with infiltration of lymphocytes, plasma cells, heterophils and macrophages in a chicken (No. S9) showed neurologic signs on day 42. (C) Diffuse proliferation of variably sized lymphoid cells among the axonal fibers in a chicken (No. S13) showed neurologic signs and MD lymphoma on day 56. (D) Moderate proliferation of lymphoid cells in a chicken (No. S18) showed neurologic signs and MD lymphoma on day 70 . Note proliferative lesions without inflammatory findings. HE stain. $\mathrm{Bar}=50 \mu \mathrm{m}$.

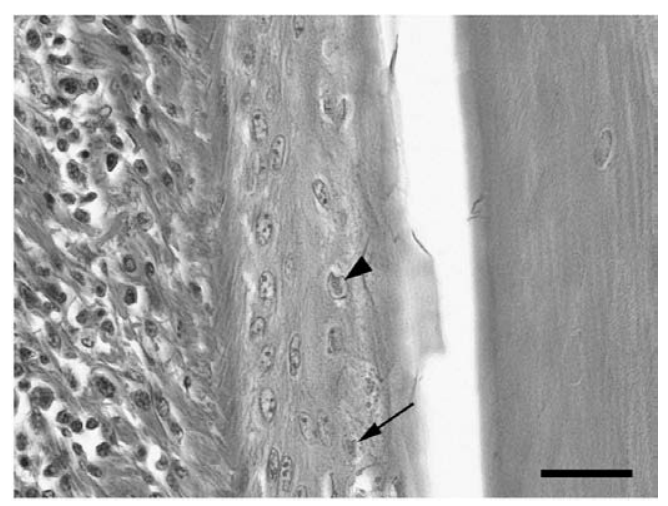

Fig. 2. MDV intranuclear (arrowhead) and intracytoplasmic inclusion bodies (arrow) on day 21 in the feather follicle epithelium of adult SPF P2 line chickens (50-week-old) inoculated with virulent MDV. Note infiltrations of lymphocytes, heterophils and macrophages around the feather follicle. HE stain. $\mathrm{Bar}=20 \mu \mathrm{m}$.

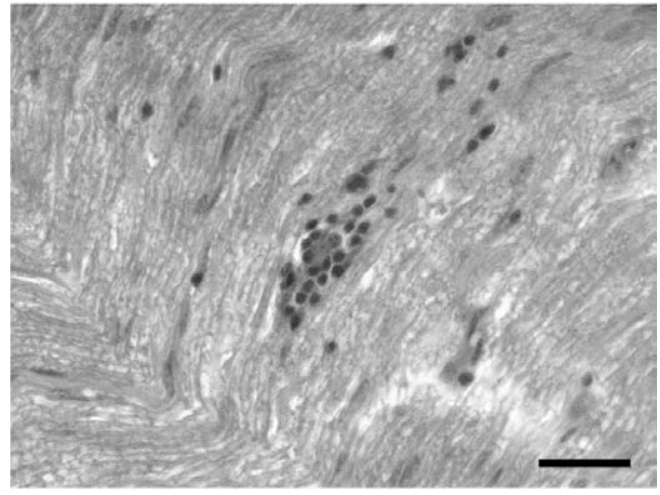

Fig. 3. Infiltrations of a small number of lymphocytes around the blood vessel in the peripheral nerve on day 70 in a commercial line chicken (74-week-old) inoculated with virulent MDV. HE stain. Bar $=20 \mu \mathrm{m}$. 

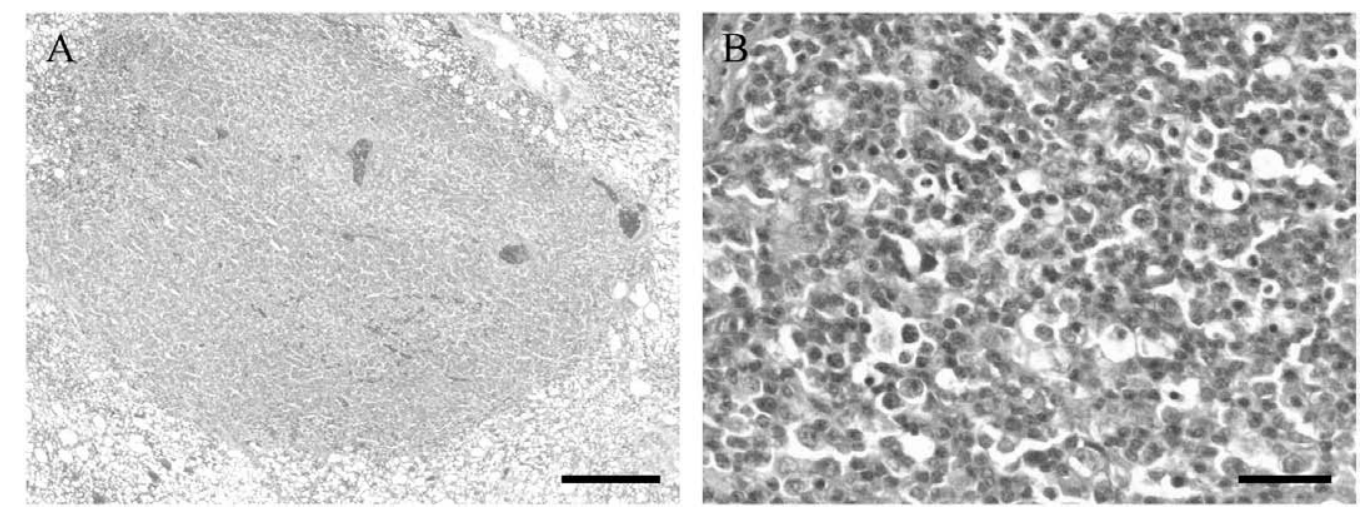

Fig. 4. A large mass of MD lymphoma constituted neoplastic lymphoid cells in the lung on day 70 in a commercial line chicken (74-week-old) inoculated with virulent MDV. (A) A large mass of neoplastic lymphoid cells suggesting MD lymphoma. Bar=50 $\mu \mathrm{m}$. (B) Variably sized lymphoid cells suggesting MD lymphoma. HE stain. Bar=20 $\mu \mathrm{m}$.

development in adult chickens. In the feather pulp, moderate to diffuse proliferation of lymphoid cells was observed in five chickens (Nos. S10 to S13, and S18). Three (Nos. $\mathrm{S} 12, \mathrm{~S} 13$ and S18) of five chickens showed visceral lymphomas, while the remaining two (Nos. S10 and S11) showed no lesions. The lesions in the feather pulp seemed to be associated with visceral MD lymphoma, although the number of affected chickens was small. The incidence of $\mathrm{MD}$ is reported to be closely related to the dynamics of inclusion body formation and feather pulp lesions in chicks $[13,14]$. The histopathological lesions of this study were also resembled which in younger chicks inoculated with KS strain $[9,18]$. However, in younger chicks, the incidence of MD was higher, and the onset of tumor formation was early noted.

In commercial line chickens, although one chicken showed lymphoma on day 70, most chickens were refractory to challenge with MDV. These results would be attributed to their genetically resistance back ground and immunity induced by MD vaccines.

There are two alternative hypothesis concerning pathogenesis of late MD, "new infection theory" and "old infection theory" [21]. New infection theory is that adult chickens have susceptibility to de novo challenge with $\mathrm{MDV}$, and newly infection induces late MD. Meanwhile, old infection theory is that latent infection MDV which triggered or exacerbated by several factors like immunodepression induces late MD. The incidence of MD in P2 line chickens would be comparable to new infection theory. The pathogenesis of one commercial line chicken developing $\mathrm{MD}$ is questionable. This chicken was in good clinical conditions throughout the experiment, and showed no histopathological immunosuppressive changes. Because MDV persisted in the chickens for a long period of time, it was not determined whether the onset of MD was caused by the inoculation or previous infection with MDV in field flocks. Further investigations for late MD in adult chickens are needed to reveal its pathogenesis. However, our model would become a tool for elucidating the etiology and pathogenesis of late MD in the field flocks.

In conclusion, this study demonstrated that adult SPF P2 line chickens are susceptible to virulent MDV, and would be useful as a late MD model.

\section{REFERENCES}

1. Anderson, D. P., Eidson, C. S. and Richey, D. J. 1971. Age susceptibility of chickens to Marek's disease. Am. J. Vet. Res. 32: 935-938.

2. Baaten, B. J., Butter, C. and Davison, T. F. 2004. Study of host-pathogen interactions to identify sustainable vaccine strategies to Marek's disease. Vet. Immuno. Immunopathol. 100: $165-177$.

3. Calnek, B. W. 2001. Pathogenesis of Marek's disease virus infection. pp. 25-56. In: Current Topics in Microbiology and Immunology (Hirai, K. ed.), Springer-Verlag, Berlin.

4. Calnek, B. W. 1973. Influence of age at exposure on the pathogenesis of Marek's disease. J. Natl. Cancer Inst. 51: 929-939.

5. Davison, F. and Kaiser, P. 2004. Immunity to Marek's disease. pp. 126-141. In: Marek's Disease: An Evolving Problem (Davison, F. and Nair, V. eds.), Elsevier Academic Press, London.

6. Davidson, I. and Borenshtain, R. 2002. The feather tips of commercial chickens are a favorable source of DNA for the amplification of Marek's disease virus and avian leukosis virus, subgroup J. Avian pathol. 31: 237-240.

7. Fehler, F. and Winter, C. 2001. CIAV infection in older chickens: an apathogenic infection? pp. 391-394. In: Proceedings of 2nd International Symposium on Infectious Bursal Disease and Chicken Anemia, Institut für Geflugelkrankheiten, Justus Liebig University, Rauischholzhausen, Giessen.

8. Gimeno, I. M., Witter, R. L. and Reed, W. M. 1999. Four distinct neurologic syndromes in Marek's disease: effect of viral strain and pathotype. Avian Dis. 43: 721-737.

9. Goryo, M., Tsukui, M., Saijo, K., Takeuchi, J., Obata, F. and Fujikawa, Y. 1980. Studies on a virulent Marek's disease virus (CVI988 strain). pp 17-30. In: English Summaries of the Studies on Poultry Disease in Japan, The Japanese Veterinary Poultry Association, Tsukuba. 
10. Japanese Association for Laboratory Animal Science. 1987. Guidelines for animal experimentation. Exp. Anim. 3: 285-288.

11. Kreager, K. S. 1997. Marek's disease: clinical aspects and current field problems in layer chickens. pp. 23-26. In: Diagnosis and Control of Neoplastic Disease of Poultry (Fadly, A. M., Schat, K. A. and Spencer, J. L. eds.), American Association of Avian Pathologists Inc., Kennett Square.

12. Lucio-Martinez, B. 1999. Impact of vv Marek's disease on mortality and production in a multiple-age farm. pp. 55-56. In: Proceedings of the 48th Western Poultry Disease Conference, Vancouver.

13. Moriguchi, R. and Izawa, H. 1979. Marek's disease in chickens: correlation of Marek's disease with nuclear-inclusion formation in feather-follicle epithelium. Avian Dis. 23: 547-554.

14. Moriguchi, R., Fujimoto, Y. and Izawa, H. 1982. Chronological observations of feather pulp lesions in chickens inoculated with Marek's disease virus. Avian Dis. 26: 375-388.

15. Morimura, T., Ohashi, K., Sugimoto, C. and Onuma, M. 1998. Pathogenesis of Marek's disease (MD) and possible mechanisms of immunity induced by MD vaccine. J. Vet. Med. Sci. 60: $1-8$.

16. Morrow, C. and Fehler, F. 2004. Marek's disease: a worldwide problem. pp. 49-61. In: Marek's Disease: An Evolving Problem (Davison, F. and Nair, V. eds.), Elsevier Academic Press, London.
17. Payne, L. N. Pathological responses to infection. 2004. pp. 7897. In: Marek's Disease: An Evolving Problem (Davison, F. and Nair, V. eds.), Elsevier Academic Press, London.

18. Saijo, K., Takeuchi, J., Goryo, M., Tamura, S., Obata, F. and Fujikawa, Y. 1980. Biological characteristics of a virulent Marek's disease virus strain isolated from the affected chicken of the field flock. pp. 23-24. In: English Summaries of the Studies on Poultry Disease in Japan, The Japanese Veterinary Poultry Association, Tsukuba.

19. Schat, K. A. 2004. Marek's disease immunosuppression. pp. 142-155. In: Marek's Disease: An Evolving Problem (Davison, F. and Nair, V. eds.), Elsevier Academic Press, London.

20. Sharma, J. M., Witter, R. L. and Burmerster, B. R. 1973. Pathogenesis of Marek's disease in old chickens: lesion regression as the basis for age-related resistance. Infect. Immun. 8: 715-724.

21. Witter, R. L. 2001. Protective efficacy of Marek's disease vaccines. pp. 58-90. In: Current Topics in Microbiology and Immunology (Hirai, K. ed.), Springer-Verlag, Berlin.

22. Witter, R. L. and Gimeno, I. M. 2006. Susceptibility of adult chickens, with and without prior vaccination, to challenge with Marek's disease virus. Avian Dis. 50: 354-365.

23. Witter, R. L., Sharma, J. M., Solomon, J. J. and Champion, L. R. 1973. An age-related resistance of chickens to Marek's disease: some preliminary observations. Avian Pathol. 2: 43-54. 